\title{
Lower starting dose of afatinib for the treatment of metastatic lung adenocarcinoma harboring exon 21 and exon 19 mutations
}

\author{
Yi-Chieh Chen 1,2, Ming-Ju Tsai ${ }^{2,3,4}$, Mei-Hsuan Lee ${ }^{3}$, Chia-Yu Kuo ${ }^{3,5}$, Mei-Chiou Shen ${ }^{6}$, Ying-Ming Tsai, ${ }^{2,3,4}$,
} Huang-Chi Chen ${ }^{5}$, Jen-Yu Hung ${ }^{3,4}$, Ming-Shyan Huang ${ }^{7}$, Inn-Wen Chong ${ }^{3,8}$ and Chih-Jen Yang ${ }^{2,3,4,8,9^{*}}$ (D)

\begin{abstract}
Background: Afatinib has shown favorable response rates (RRs) and longer progression free survival (PFS) in lung cancer patients harboring EGFR mutations compared with standard platinum-based chemotherapy. However, serious adverse drug reactions (ADRs) limit the clinical application of afatinib.

Methods: We designed a retrospective study, enrolling all patients with metastatic lung adenocarcinoma who were diagnosed and treated with 30 or 40 mg daily afatinib as their initial treatment in three Kaohsiung Medical University-affiliated hospitals in Taiwan.

Results: A total of 179 patients were enrolled in the study, of which 102 (57\%) and 77 (43\%) received $30 \mathrm{mg}$ and $40 \mathrm{mg}$ afatinib daily as their initial treatment, respectively. The patients initially using $30 \mathrm{mg}$ afatinib daily had a similar RR (75\% vs. 83\%, $p=0.1672$ ), median PFS (14.5 vs. 14.8 months, log-rank $p=0.4649)$, and median OS (34.0 vs. 25.2 months, log-rank $p=0.5982$ ) compared with those initially using $40 \mathrm{mg}$ afatinib daily. Patients initially receiving $30 \mathrm{mg}$ afatinib daily had fewer ADRs compared with those using $40 \mathrm{mg}$ daily. The overall incidence of moderate and severe ADRs was significantly lower in patients receiving $30 \mathrm{mg}$ afatinib daily compared with those using 40 mg daily (49\% vs. 77\%, $p=0.002)$; similar findings was observed in terms of severe ADRs (7\% vs. 24\%, $p<0.0001$ ).
\end{abstract}

Conclusion: Patients receiving $30 \mathrm{mg}$ afatinib daily as their initial treatment had similar RR, PFS, OS, but significantly fewer serious ADRs, as compared with those using $40 \mathrm{mg}$ as their starting dose.

Keywords: Epidermal growth factor receptor tyrosine kinase inhibitor, Afatinib, Lower starting dose, Adverse drug reaction

\footnotetext{
* Correspondence: chjeya@cc.kmu.edu.tw

${ }^{2}$ Graduate Institute of Medicine, College of Medicine, Kaohsiung Medical University, Kaohsiung, Taiwan

${ }^{3}$ Division of Pulmonary and Critical Care Medicine, Kaohsiung Medical University Hospital, Kaohsiung Medical University, Kaohsiung, Taiwan

Full list of author information is available at the end of the article
}

(c) The Author(s). 2021 Open Access This article is licensed under a Creative Commons Attribution 4.0 International License, which permits use, sharing, adaptation, distribution and reproduction in any medium or format, as long as you give appropriate credit to the original author(s) and the source, provide a link to the Creative Commons licence, and indicate if changes were made. The images or other third party material in this article are included in the article's Creative Commons licence, unless indicated otherwise in a credit line to the material. If material is not included in the article's Creative Commons licence and your intended use is not permitted by statutory regulation or exceeds the permitted use, you will need to obtain permission directly from the copyright holder. To view a copy of this licence, visit http://creativecommons.org/licenses/by/4.0/ The Creative Commons Public Domain Dedication waiver (http://creativecommons.org/publicdomain/zero/1.0/) applies to the data made available in this article, unless otherwise stated in a credit line to the data. 


\section{Highlights}

1. The patients initially using $30 \mathrm{mg}$ afatinib daily had a similar RR (75\% vs. $83 \%, p=0.1672)$, median PFS (14.5 vs. 14.8 months, $\log$-rank $p=0.4649$ ), and median OS (34.0 vs. 25.2 months, log-rank $p=0.5982$ ) compared with those initially using $40 \mathrm{mg}$ afatinib daily.

2. Patients with a lower starting dose had fewer ADRs including diarrhea, stomatitis, dry skin, acne and/or skin rash, and pruritis compared with patients receiving $40 \mathrm{mg}$ as their starting dose. The overall incidence of grade 3 ADRs was significantly lower in patients receiving $30 \mathrm{mg}$ afatinib daily compared with those receiving $40 \mathrm{mg}$ ( $7 \%$ vs $24 \%, p<0.0001)$.

3. Serious adverse drug reactions (ADRs) may limit the clinical application of a higher dose of afatinib ( $40 \mathrm{mg}$ daily) because about $40 \%$ of them have to discontinue their treatment or reduce the dosage due to severe ADRs.

\section{Background}

Lung cancer is a leading cause of cancer related mortality worldwide, including in Taiwan. Most lung cancer patients are diagnosed at an advanced stage meaning salvage therapy is recommended [1]. Platinum-based chemotherapy is a standard therapy for advanced stage lung cancer but has only been proven to have modest clinical efficacy [2, 3]; the response rates (RRs) to 1st line cytotoxic chemotherapy for advanced non-small cell lung cancer (NSCLC) are 30 to $40 \%$, and all patients eventually develop resistance with a median survival of only 8 to 10 months [3].

In addition, chemotherapy causes a number of severe adverse drug reactions (ADRs), such as nausea, vomiting, hematological toxicity and some unexpected lifethreatening complications [3, 4], which can cause poor quality of life. Therefore, many new treatment strategies have been developed to improve the clinical efficacy of chemotherapy and to lower its toxicity. Driver mutations are believed to be involved in cancer pathogenesis and small molecular drugs designed to target the signal transduction pathway can result in cell apoptosis or death; these are often accompanied by fewer ADRs than standard chemotherapy $[5,6]$.

Therefore, many new target therapies were developed which have been proven to have better clinical efficacy compared with standard platinum-based chemotherapy [5]. Several large-scale phase 3 clinical trials have shown that lung cancer patients harboring susceptible epidermal growth factor receptor (EGFR) mutations who received an EGFR tyrosine kinase inhibitor (TKI) have better clinical efficacy compared with platinum-based chemotherapy, in terms of overall RRs, progression free survival (PFS) and quality of life [7-11]. Afatinib is an irreversible, second-generation EGFR TKI [12] which has been shown to have better RR, PFS and overall survival (OS) when used in patients of lung cancer harboring susceptible EGFR mutations, compared with platinumbased chemotherapy [13, 14]. Furthermore, afatinib has been shown to have a significantly longer PFS and timeto-treatment failure compared with gefitinib when used as the initial EGFR TKI in a head-to-head phase 2B clinical trial;. Afatinib also has been proven to have significantly longer OS in patients of lung cancer with exon 19 deletions [15]. Therefore, afatinib is a promising EGFR TKI for the management of patients with lung cancer with EGFR mutations.

However, ADRs were reported in $11 \%$ of patients taking $40 \mathrm{mg}$ afatinib daily and $4 \%$ of patients taking gefitinib [16]. A meta-analysis showed that in patients who received first- and second-generation EGFR TKIs, 40\% experienced grade 3-4 ADRs, while the risk of grade 34 ADRs was lower for gefitinib (29.1\%) than for erlotinib (54.1\%) or afatinib (42.1\%) [6] . Another pooled safety study concluded that grade 3-4 skin rash and diarrhea occurred significantly more frequently with afatinib therapy compared with erlotinib or gefitinib therapy [17]. Patients receiving afatinib treatment always have more ADRs compared with 1st generation EGFR TKIs in patients with EGFR mutations, and 28 to $53.3 \%$ of patients receiving standard $40 \mathrm{mg}$ afatinib daily had to discontinue or reduce their dose due to severe ADRs in the phase 3 LUX-Lung 3 and LUX-Lung 6 trials [13, 14, 18]. Real-world data of 1st-line afatinib treatment showed that dose reduction occurred in 47.5 to $76.3 \%$ of cases $[19,20]$. Dose reductions were mainly due to ADRs and were more common in females, East-Asian individuals and those with a lower body weight [21]. Therefore, methods for ameliorating ADRs whilst maintaining clinical efficacy are urgently needed for lung cancer patients receiving afatinib as their first-line therapy. A lower starting dose of afatinib was tested by clinicians in clinical practice and several trials [22]. In May 2014, the Taiwan Nation Health Insurance Bureau permitted both $30 \mathrm{mg}$ and $40 \mathrm{mg}$ afatinib daily as a first-line therapy for advanced lung adenocarcinoma with susceptible EGFR mutations.

Our preliminary report, a very small-scale study that only enrolled 48 patients with different starting doses, showed that patients who received $30 \mathrm{mg}$ afatinib daily as the starting dose had non-inferior PFS with fewer severe ADRs [23]. We believe that fewer adverse events, especially fewer severe ADRs will result in good drug compliance and a better quality of life during lung cancer treatment.

Herein, we designed a larger-scale retrospective study to investigate whether patients of lung adenocarcinoma 
with susceptible EGFR mutations receiving a lower starting doses of afatinib had a similar clinical effectiveness and fewer severe ADRs compared with those taking a higher starting dose of afatinib in Taiwan.

\section{Methods}

\section{Patient identification}

Patients with metastatic lung adenocarcinoma who were diagnosed and treated between May 1st 2014 and July 31st 2019 in Kaohsiung Medical University Hospital (KMUH), Kaohsiung Municipal Ta-Tung Hospital and Kaohsiung Municipal Siaogang Hospital (all Kaohsiung Medical University-affiliated hospitals) in Taiwan, were identified and followed until Dec 31st, 2019. The diagnosis of lung adenocarcinoma was confirmed pathologically according to the World Health Organization pathology classification. Tumor staging was assessed according to the Seventh American Joint Committee Cancer Staging System and confirmed by a multidisciplinary lung cancer team. All adenocarcinoma specimens were analyzed using an EGFR RGQ kit (Qiagen, UK). The protocol was developed and validated by the Division of Molecular Diagnostics, Department of Laboratory Medicine, $\mathrm{KMUH}$, and utilized amplification refractory mutation specific (ARMS) polymerase chain reactions (PCRs) and Scorpion technologies for detection; direct sequencing was performed if a negative result was found in the ARMS PCR. The examination techniques were consistent with our previous studies [23-27].

In the current study, we enrolled all individuals with exon 19 deletions and exon 21 L858R point mutations, and excluded those with resistant mutation; they were all treatment-naïve and were treated with either 30 or $40 \mathrm{mg}$ afatinib daily as their first-line treatment for stage IV metastatic lung adenocarcinoma. Baseline clinical characteristics were determined by retrospective chart review, including age at diagnosis, sex, weight, height, Eastern Cooperative Oncology Group (ECOG) performance status, glomerular filtration rate, smoking history, hepatitis $\mathrm{B}$, hepatitis $\mathrm{C}$, tuberculosis history, family history, thyroid transcription factor-1 (TTF-1) stain, programmed death-ligand 1 (PDL-1) stain, EGFR mutation, TNM status, and number of metastatic sites/organs on initial diagnosis.

The initial treatment response was classified based on serial imaging studies using the revised Response Evaluation Criteria in Solid Tumors (RECIST 1.1) criteria. The PFS and OS of the first-line afatinib treatment were defined as the time from the start of the first treatment to the date of disease progression on an imaging examination, and the date of death, respectively. ADRs were recorded by physicians and graded according to the Common Terminology Criteria for Adverse Events version 4.0.

\section{Statistical analysis}

Categorical variables and continuous variables were compared using $X^{2}$ test and Student's t-test, respectively. Survival times were estimated using the Kaplan-Meier method, with differences between the two groups compared using the log-rank test. Univariate Cox regression analyses were performed to identify the factors associated with PFS and OS. Using a backward variable selection method, keeping only variables with $p$ values $<0.1$, we developed reduced multivariable models with Cox regression analyses to determine independent predictive factors for PFS and OS. Hazard ratios (HR) with 95\% confidence intervals (CIs) of the factors are reported. All statistical analyses were performed using SAS software (version 9.4 for Windows, SAS Institute Inc., Cary, NC, USA). Statistical significance was set at a two-sided $p$ value of $<0.05$.

\section{Results}

During the study period, a total of 179 patients with stage IV lung adenocarcinoma harboring exon 19 deletions or an exon $21 L 858 R$ point mutation who received afatinib as their first-line therapy were enrolled (Table 1). Of these patients, $102(57 \%)$ received $30 \mathrm{mg}$ afatinib daily and $77(43 \%)$ received $40 \mathrm{mg}$ daily as their initial treatment. Patients receiving $30 \mathrm{mg}$ daily as their initial dose, compared with those receiving $40 \mathrm{mg}$ afatinib daily, were significantly older $(65.7 \pm 9.3$ vs. $62.4 \pm 9.3$ years, $p=0.0199)$, had a significantly lower weight $(58.2 \pm 12.5$ vs. $62.4 \pm 9.9 \mathrm{~kg}, p=0.014)$ and a significantly lower body surface area $\left(1.6 \pm 0.2\right.$ vs. $\left.1.7 \pm 0.2 \mathrm{~m}^{2} p=0.0078\right)$, and were more likely to be female ( $72 \%$ vs. $48 \%)$. Patients in both groups has similar number of metastatic sites at the initial diagnosis $(p=0.2360)$, while significantly more patients with brain metastasis received $40 \mathrm{mg}$ daily of afatinib as their initial dose, rather than $30 \mathrm{mg}$ daily ( $42 \%$ vs. $21 \%, p=0.0023)$. There was no significant difference in terms of other metastatic sites. There were no significant differences in body height, smoking history, glomerular filtration rate, serum albumin level, serum levels of liver enzymes, tuberculosis history, family history, performance status, TTF-1 staining, PDL-1 staining, and the EGFR gene mutation site (exon 19 or 21) of the cancer specimens between the two groups.

\section{Outcomes for $\mathbf{3 0 ~} \mathrm{mg}$ and $\mathbf{4 0} \mathrm{mg}$ afatinib daily as the first- line treatment}

Patients initially receiving $30 \mathrm{mg}$ afatinib daily had similar response rates $(75 \%$ vs. $83 \% ; p=0.1672)$ and similar disease control rates (99\% vs. $96 \%$ ) compared with those initially receiving $40 \mathrm{mg}$ afatinib daily (Table 2 ). The PFS was not significantly different between patients receiving $30 \mathrm{mg}$ and $40 \mathrm{mg}$ afatinib daily (median PFS: 14.5 vs. 14.8 months, $\log$-rank $p=0.4649$; Fig. 1a). In terms of 
Table 1 Clinical characteristics and treatment responses for all patients

\begin{tabular}{|c|c|c|c|c|}
\hline Variables & All patients & Afatinib $30 \mathrm{mg}$ daily & Afatinib $40 \mathrm{mg}$ daily & $P$ value \\
\hline $\mathrm{N}$ & 179 & 102 & 77 & \\
\hline Age (year) & $64.3 \pm 9.4$ & $65.7 \pm 9.3$ & $62.4 \pm 9.3$ & 0.0199 \\
\hline Age group: & & & & 0.0212 \\
\hline$<65$ years old & $80(45 \%)$ & $38(37 \%)$ & $42(55 \%)$ & \\
\hline$\geq 65$ years old & $99(55 \%)$ & $64(63 \%)$ & $35(45 \%)$ & \\
\hline Sex & & & & 0.0014 \\
\hline Female & 110 (61\%) & $73(72 \%)$ & $37(48 \%)$ & \\
\hline Male & $69(39 \%)$ & $29(28 \%)$ & $40(52 \%)$ & \\
\hline Smoking history: & & & & 0.9349 \\
\hline Never smoker & $140(78 \%)$ & $80(78 \%)$ & $60(78 \%)$ & \\
\hline Ever smoker & $39(22 \%)$ & $22(22 \%)$ & $17(22 \%)$ & \\
\hline Height (cm) & $160 \pm 8$ & $159.1 \pm 7.7$ & $161.2 \pm 8.4$ & 0.0905 \\
\hline Weight (kg) & $60 \pm 11.6$ & $58.2 \pm 12.5$ & $62.4 \pm 9.9$ & 0.0140 \\
\hline Body mass index $\left(\mathrm{kg} / \mathrm{m}^{2}\right)$ & $23.3 \pm 3.6$ & $22.8 \pm 3.7$ & $24 \pm 3.4$ & 0.0339 \\
\hline Body surface area $\left(\mathrm{m}^{2}\right)$ & $1.6 \pm 0.2$ & $1.6 \pm 0.2$ & $1.7 \pm 0.2$ & 0.0078 \\
\hline Serum creatinine level & $0.8 \pm 0.3$ & $0.8 \pm 0.3$ & $0.8 \pm 0.3$ & 0.4561 \\
\hline eCCr-CG $(\mathrm{mL} / \mathrm{min})^{\mathrm{a}}$ & $77.1 \pm 26.8$ & $73.7 \pm 25.9$ & $81.5 \pm 27.5$ & 0.0566 \\
\hline eGFR-MDRD $\left(\mathrm{mL} / \mathrm{min} / 1.73 \mathrm{~m}^{2}\right)^{\mathrm{b}}$ & $88.8 \pm 27$ & $88 \pm 26.8$ & $89.8 \pm 27.4$ & 0.6628 \\
\hline Serum albumin (mg/dL) & $4.0 \pm 0.5$ & $4.0 \pm 0.4$ & $4.1 \pm 0.5$ & 0.2751 \\
\hline Serum glutamic oxaloacetic transaminase (U/L) & $29.6 \pm 22.8$ & $31.4 \pm 28.7$ & $27.3 \pm 10.4$ & 0.1835 \\
\hline Serum glutamic pyruvic transaminase $(U / L)$ & $26.5 \pm 25.1$ & $26.7 \pm 28.5$ & $26.3 \pm 20$ & 0.9178 \\
\hline Hepatitis B: ${ }^{c}$ & & & & 0.4999 \\
\hline Negative & $147(84 \%)$ & $86(85 \%)$ & $61(81 \%)$ & \\
\hline Positive & $29(16 \%)$ & $15(15 \%)$ & $14(19 \%)$ & \\
\hline Hepatitis C: ${ }^{c}$ & & & & 0.0370 \\
\hline Negative & $165(94 \%)$ & $98(97 \%)$ & $67(89 \%)$ & \\
\hline Positive & $11(6 \%)$ & $3(3 \%)$ & $8(11 \%)$ & \\
\hline Old tuberculosis: & & & & 0.8901 \\
\hline Negative & $174(97 \%)$ & 99 (97\%) & 75 (97\%) & \\
\hline Positive & $5(3 \%)$ & $3(3 \%)$ & $2(3 \%)$ & \\
\hline Family history: & & & & 0.0859 \\
\hline Negative & $168(94 \%)$ & $93(91 \%)$ & 75 (97\%) & \\
\hline Positive & $11(6 \%)$ & $9(9 \%)$ & $2(3 \%)$ & \\
\hline Performance status while starting afatinib: & & & & 0.1024 \\
\hline $\mathrm{ECOG} \leq 1$ & $146(82 \%)$ & 79 (77\%) & $67(87 \%)$ & \\
\hline$E C O G \geq 2$ & $33(18 \%)$ & $23(23 \%)$ & $10(13 \%)$ & \\
\hline TTF-1 stain: ${ }^{c}$ & & & & 0.8901 \\
\hline Positive & $170(100 \%)$ & 99 (100\%) & $71(100 \%)$ & \\
\hline PDL-1 stain: ${ }^{c}$ & & & & 0.4732 \\
\hline Absence & $33(38 \%)$ & $16(34 \%)$ & $17(41 \%)$ & \\
\hline Presence & $55(63 \%)$ & $31(66 \%)$ & $24(59 \%)$ & \\
\hline \multicolumn{5}{|l|}{ EGFR gene mutation site: ${ }^{d}$} \\
\hline Exon 19 & $95(53 \%)$ & $60(59 \%)$ & $35(45 \%)$ & 0.0760 \\
\hline Exon 21 & $85(47 \%)$ & $42(41 \%)$ & $43(56 \%)$ & 0.0517 \\
\hline
\end{tabular}


Table 1 Clinical characteristics and treatment responses for all patients (Continued)

\begin{tabular}{|c|c|c|c|c|}
\hline Variables & All patients & Afatinib $30 \mathrm{mg}$ daily & Afatinib $40 \mathrm{mg}$ daily & $P$ value \\
\hline \multicolumn{5}{|l|}{ TNM staging: } \\
\hline N2-3 & $112(63 \%)$ & $67(66 \%)$ & $45(58 \%)$ & 0.3214 \\
\hline M1a-c & $179(100 \%)$ & $102(100 \%)$ & $77(100 \%)$ & 0.5136 \\
\hline Number of metastatic sites/organs (1-2 vs. $>2$ ) & & & & 0.2360 \\
\hline 1 site & $64(36 \%)$ & $41(40 \%)$ & $23(30 \%)$ & \\
\hline 2 sites & $69(39 \%)$ & $39(38 \%)$ & $30(39 \%)$ & \\
\hline$\geq 3$ sites & $46(26 \%)$ & $22(22 \%)$ & $24(31 \%)$ & \\
\hline \multicolumn{5}{|l|}{ Metastatic site/organ on initial diagnosis } \\
\hline Brain & $53(30 \%)$ & $21(21 \%)$ & $32(42 \%)$ & 0.0023 \\
\hline Lung & $79(44 \%)$ & $40(39 \%)$ & $39(51 \%)$ & 0.1272 \\
\hline Pleura (or with pleural effusion) & $80(45 \%)$ & $47(46 \%)$ & $33(43 \%)$ & 0.6678 \\
\hline Bone & $99(55 \%)$ & $59(58 \%)$ & 40 (52\%) & 0.4322 \\
\hline Liver & $22(12 \%)$ & 15 (15\%) & 7 (9\%) & 0.2573 \\
\hline Adrenal & $16(9 \%)$ & $8(8 \%)$ & $8(10 \%)$ & 0.5544 \\
\hline Other sites & $11(6 \%)$ & $5(5 \%)$ & $6(8 \%)$ & 0.4253 \\
\hline
\end{tabular}

Data are presented in mean \pm standard deviation (SD) or $\mathrm{n}(\%)$

${ }^{a} e C C r$-CG estimated creatinine clearance rate using Cockcroft-Gault equation, while $\mathrm{CrCl}$ was multiplied by 0.85 for female patients

${ }^{\mathrm{b}}$ eGFR-MDRD estimated glomerular filtration rate using Modification of Diet in Renal Disease equation. GFR was multiplied by 0.742 and 1.212 for female patients and African-American, respectively

'Including missing values

${ }^{\mathrm{d}} \mathrm{A}$ patient had EGFR mutation in both exon 19 and exon 21

OS, there was no significant difference between the two patient groups (median OS: 34.0 months vs. 25.2 months, log-rank $p=0.5982$; Fig. 1b). Notable, only 13 patients (13\%) receiving $30 \mathrm{mg}$ afatinib and 5 patients (6\%) receiving $40 \mathrm{mg}$ afatinib daily as their initial treatment received osimertinib after developing acquired resistance to afatinib. Other patients chose to have platinum-based chemotherapy or hospice care as their second-line management.

To identify factors associated with PFS, we built several Cox regression models (Table 3 ). In the univariate analyses, only the number of metastatic sites $\geq 3$, pleural metastasis (or with pleural effusion), and bone metastasis were significantly risk factors for worse PFS, while dose reduction was associated with better PFS. In the model 1 of multivariable analysis, we found two independent predicting factors for PFS, higher number of metastatic sites $(\geq 3)$ (HR [95\% CI]: 1.83 [1.19-2.84], $p=0.0065)$ and dose reduction (HR [95\% CI]: 0.57 [0.33-0.99], $p=$ $0.0467)$. In the model 2 of multivariable analysis, considering the detailed metastatic sites instead of number of metastatic sites, we found exon 21 (HR [95\% CI]: 1.56 [1.04-2.36], $p=0.0336$ ), pleural metastasis/effusion (HR [95\% CI]: 1.77 [1.17-2.69], $p=0.0075)$, and bone metastasis (HR [95\% CI]: 1.69 [1.13-2.55], $p=0.0116)$ were independent risk factors for poorer PFS, while dose reduction showed a trend toward better PFS (HR [95\% $\mathrm{CI}]: 0.59$ [0.34-1.03], $p=0.0640$ ).

We also built several Cox regression models (Table 4) to identify factors associated with OS. In the univariate analyses, male, smoking history, poorer performance status (ECOG $\geq 2$ ) were significant risk factors associated with poorer OS. The model 1 of multivariable analysis showed that male (HR [95\% CI]: 1.97 [1.21-3.22], $p=0.0066)$ and

Table 2 Initial treatment response to different initial afatinib doses

\begin{tabular}{|c|c|c|c|c|}
\hline Variables & All patients & Afatinib 30 mg daily & Afatinib $40 \mathrm{mg}$ daily & $P$ value \\
\hline Initial response to afatinib treatment $-\mathrm{n}(\%)$ & & & & 0.1661 \\
\hline Complete response & $2(1 \%)$ & $1(1 \%)$ & $1(1 \%)$ & \\
\hline Partial response & $138(77 \%)$ & $75(74 \%)$ & $63(82 \%)$ & \\
\hline Stable disease & $35(20 \%)$ & $25(25 \%)$ & $10(13 \%)$ & \\
\hline Progressive disease & $4(2 \%)$ & $1(1 \%)$ & $3(4 \%)$ & \\
\hline Disease control rate with afatinib treatment (\%) & $175(98 \%)$ & 101 (99\%) & $74(96 \%)$ & 0.1913 \\
\hline Response rate with afatinib treatment (\%) & $140(78 \%)$ & $76(75 \%)$ & $64(83 \%)$ & 0.1672 \\
\hline
\end{tabular}



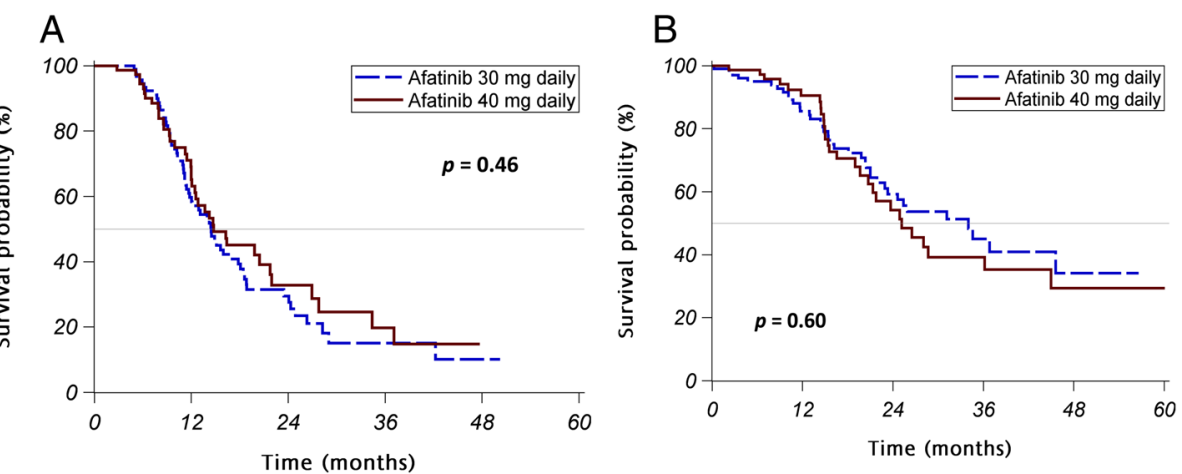

Fig. 1 PFS and OS for afatinib treatment. a PFS in patients receiving different initial doses of afatinib. $\mathbf{b}$ OS for patients receiving different initial doses of afatinib

poorer performance status (HR [95\% CI]: 2.28 [1.35-3.85], $p=0.0021$ ) were independently associated with poorer OS, while the higher number of metastatic sites $(\geq 3)$ was only associated with a trend toward poorer OS (HR [95\% CI]: 1.61 [0.96-2.68], $p=0.0709)$. In the model 2 of multivariable analyses, we found three independent predicting risk factors for OS, including smoking history (HR [95\% CI]: 2.37 [1.35-4.17], $p=0.0028$ ), poorer performance status (HR [95\% CI]: 3.00 [1.74-5.15], $p<0.0001$ ), bone metastasis (HR [95\% CI]: 1.73 [1.04-2.87], $p=0.0341$ ), while adrenal gland metastasis was associated with better OS (HR [95\% CI]: 0.33 [0.12-0.92], $p=0.0338$ ).

\section{ADRs for patients using $30 \mathrm{mg}$ or $\mathbf{4 0} \mathrm{mg}$ afatinib daily as the first-line treatment}

The most common ADRs in patients taking afatinib included acne and/or skin rash (81\%), diarrhea (74\%), dry skin (61\%), and paronychia (51\%) (Table 5). Patients receiving $30 \mathrm{mg}$ afatinib daily had a lower incidence of diarrhea (68\% vs. $82 \%)$, acne and/or skin rash ( $78 \%$ vs. $84 \%)$, dry skin ( $60 \%$ vs. $64 \%$ ) and pruritis ( $23 \%$ vs $43 \%)$ compared with those receiving $40 \mathrm{mg}$ afatinib daily. In terms of the maximal grade of ADRs, patients receiving $30 \mathrm{mg}$ daily had less severe events than those receiving $40 \mathrm{mg}$ daily $(p<0.0001)$. The patients receiving $30 \mathrm{mg}$ afatinib daily initially, compared than those taking $40 \mathrm{mg}$ afatinib daily, had a significantly lower overall incidence of moderate and severe (at least grade 2) (49\% vs. $77 \%$, $p=0.0002)$ and severe (at least grade 3$)(7 \%$ vs. $24 \%$, $p<0.0001)$ adverse drug reactions, particularly in diarrhea and adverse events involving skin. The incidences of drug-induced hepatitis and interstitial lung disease were very low and no significant differences were observed between the $30 \mathrm{mg}$ and $40 \mathrm{mg}$ groups in the present retrospective study.

More patients receiving initial afatinib dose of $40 \mathrm{mg}$ daily required dose reduction (or discontinuation) than

Table 3 Cox regression analyses to identify the factors associated with progression-free survival (PFS)

\begin{tabular}{|c|c|c|c|c|c|c|}
\hline \multirow[t]{2}{*}{ Variables } & \multicolumn{2}{|c|}{ Univariate analysis } & \multicolumn{2}{|c|}{ Multivariable analysis - model $1^{a}$} & \multicolumn{2}{|c|}{ Multivariable analysis - model $2^{a}$} \\
\hline & $\mathrm{HR}[95 \% \mathrm{Cl}]$ & $P$ value & $\overline{\mathrm{HR}}[95 \% \mathrm{Cl}]$ & $P$ value & $\mathrm{HR}[95 \% \mathrm{Cl}]$ & $P$ value \\
\hline$\overline{\text { Afatinib dose (40 mg daily vs. } 30 \mathrm{mg} \text { daily) }}$ & $0.86[0.58-1.29]$ & 0.4657 & & & & \\
\hline Sex (male vs. female) & $1.19[0.80-1.79]$ & 0.3920 & & & & \\
\hline Age ( $\geq 65$ vs. $<65$ ) & $0.79[0.53-1.17]$ & 0.2333 & & & & \\
\hline Smoking history (ever smokers vs. never smokers) & $1.16[0.71-1.89]$ & 0.5659 & & & & \\
\hline ECOG ( $\geq 2$ vs. $\leq 1)$ & $1.11[0.67-1.84]$ & 0.6762 & & & & \\
\hline Exon 21 vs. exon $19^{b}$ & $1.31[0.89-1.95]$ & 0.1751 & & & $1.56[1.04-2.36]$ & 0.0336 \\
\hline Number of metastatic sites/organs (1-2 vs. $\geq 3$ ) & $1.86[1.20-2.88]$ & 0.0055 & $1.83[1.19-2.84]$ & 0.0065 & & \\
\hline \multicolumn{7}{|l|}{ Metastatic site/organ on initial diagnosis: (yes vs. no) } \\
\hline Brain & $0.96[0.62-1.49]$ & 0.8479 & & & & \\
\hline Lung & $0.96[0.64-1.44]$ & 0.8318 & & & & \\
\hline Pleura (or with pleural effusion) & $1.57[1.05-2.35]$ & 0.0292 & & & $1.77[1.17-2.69]$ & 0.0075 \\
\hline Bone & $1.52[1.02-2.27]$ & 0.0385 & & & $1.69[1.13-2.55]$ & 0.0116 \\
\hline Liver & $1.50[0.89-2.54]$ & 0.1266 & & & & \\
\hline Adrenal gland & $0.96[0.50-1.84]$ & 0.8955 & & & & \\
\hline Other site & $0.84[0.34-2.07]$ & 0.7055 & & & & \\
\hline Dose reduction (yes vs. no) & $0.56[0.32-0.98]$ & 0.0407 & $0.57[0.33-0.99]$ & 0.0467 & $0.59[0.34-1.03]$ & 0.0640 \\
\hline
\end{tabular}


Table 4 Cox regression analyses to identify the factors associated with overall survival (OS)

\begin{tabular}{|c|c|c|c|c|c|c|}
\hline \multirow[t]{2}{*}{ Variables } & \multicolumn{2}{|c|}{ Univariate analysis } & \multicolumn{2}{|c|}{$\underset{\mathrm{a}}{\text { Multivariable analysis - model } 1}$} & \multicolumn{2}{|c|}{$\begin{array}{l}\text { Multivariable analysis - model } 2 \\
\text { a }\end{array}$} \\
\hline & $\mathrm{HR}[95 \% \mathrm{Cl}]$ & $P$ value & $\mathrm{HR}[95 \% \mathrm{Cl}]$ & $P$ value & $\mathrm{HR}[95 \% \mathrm{Cl}]$ & $P$ value \\
\hline Afatinib dose (40 mg daily vs. $30 \mathrm{mg}$ daily) & $1.14[0.70-1.84]$ & 0.5986 & & & & \\
\hline Sex (male vs. female) & $1.97[1.22-3.20]$ & 0.0058 & $1.97[1.21-3.22]$ & 0.0066 & & \\
\hline Age ( $\geq 65$ vs. $<65)$ & $0.93[0.58-1.51]$ & 0.7729 & & & & \\
\hline Smoking history (ever smokers vs. never smokers) & $1.90[1.10-3.29]$ & 0.0210 & & & $2.37[1.35-4.17]$ & 0.0028 \\
\hline ECOG ( $\geq 2$ vs. $\leq 1)$ & $2.46[1.46-4.15]$ & 0.0007 & $2.28[1.35-3.85]$ & 0.0021 & $3.00[1.74-5.15]$ & $<0.0001$ \\
\hline Exon 21 vs. exon $19^{b}$ & $0.95[0.59-1.54]$ & 0.8334 & & & & \\
\hline Number of metastatic sites/organs (1-2 vs. $\geq 3$ ) & $1.6[0.96-2.67]$ & 0.0690 & $1.61[0.96-2.68]$ & 0.0709 & & \\
\hline \multicolumn{7}{|l|}{ Metastatic site/organ on initial diagnosis: (yes vs. no) } \\
\hline Brain & $1.54[0.93-2.55]$ & 0.0967 & & & & \\
\hline Lung & $1.08[0.66-1.76]$ & 0.7713 & & & & \\
\hline Pleura (or with pleural effusion) & $1.32[0.82-2.12]$ & 0.2584 & & & & \\
\hline Bone & $1.64[0.99-2.70]$ & 0.0532 & & & $1.73[1.04-2.87]$ & 0.0341 \\
\hline Liver & $1.28[0.68-2.39]$ & 0.4395 & & & & \\
\hline Adrenal gland & $0.45[0.16-1.25]$ & 0.1264 & & & $0.33[0.12-0.92]$ & 0.0338 \\
\hline Other site & $1.09[0.40-3.01]$ & 0.8668 & & & & \\
\hline Dose reduction (yes vs. no) & $1.08[0.61-1.92]$ & 0.7903 & & & & \\
\hline
\end{tabular}

${ }^{a}$ Multivariable Cox regression models were built using backward variable selection method, keeping only variables with $p$ values less than 0.1 . Number of metastatic sites/organs was considered while building model 1, whereas the detailed metastatic sites were considered while building model 2

${ }^{\mathrm{b}}$ The patient with mutation in both exon 21 and exon 19 was arbitrary classified in to exon 21 group

those receiving $30 \mathrm{mg}$ daily initially ( $40 \%$ vs. $8 \%, p<$ 0.0001) (Table 6).

\section{Cancer recurrence in patients using $30 \mathrm{mg}$ or $40 \mathrm{mg}$ afatinib daily as a first-line treatment}

The initial afatinib dose of $30 \mathrm{mg}$ daily and $40 \mathrm{mg}$ daily showed similar recurrence rate ( $54 \%$ vs. $45 \%, p=0.2620)$ (Table 7). In terms of the recurrent sites, patients receiving $30 \mathrm{mg}$ daily initially had a higher incidence of bone metastasis as the recurrent site compared with the 40 mg group ( $13 \%$ vs. $4 \%, p=0.0399)$. Notably, there was no significant difference in the occurrence of central nervous system (including brain or leptomeningeal) metastasis as the recurrent site between patients in the 30 $\mathrm{mg}$ and $40 \mathrm{mg}$ afatinib groups ( $18 \%$ vs. $21 \%, p=0.5969)$.

\section{Discussion}

To the best of our knowledge, this retrospective trial is the largest evaluation of patients receiving $30 \mathrm{mg}$ afatinib daily as the starting dose for the treatment of metastatic lung adenocarcinoma harboring exon 21 L858R point mutations or exon 19 deletions. We demonstrated that patients who received $30 \mathrm{mg}$ afatinib daily as their starting dose had similar RRs, PFS, and OS compared with patients who received $40 \mathrm{mg}$ daily as their starting dose, and they also had fewer severe ADRs.

The patients receiving $30 \mathrm{mg}$ afatinib daily as the initial dose tended to be older, female sex, smaller in body size (less weight, lower body mass index, and lower body surface area), compared with those starting with $40 \mathrm{mg}$ daily. These results are similar to previous studies [23, 28].

The patients initially using $30 \mathrm{mg}$ afatinib daily had similar RRs as the $40 \mathrm{mg}$ afatinib group, and the RR was comparable with previous studies (61-74\%) [29]. In two phase 3 clinical trials, LUX-Lung 3 and LUX-Lung 6, the median PFS among patients of lung adenocarcinoma harboring EGFR mutations taking $40 \mathrm{mg}$ afatinib as their initial dose was 10.9 and 13.6 months, respectively. A real-word practice study in Japan that enrolled 128 patients reported a median PFS of 17.8 months [20], while a phase 2 study which used a lower starting dose of 20 mg daily afatinib that increased in 10-mg increments up to $50 \mathrm{mg}$ daily, reported a PFS of 15.2 months [22]. Another phase 2 study that enrolled 40 elderly patients had a shorter PFS of 12.9 months [19]. The current study revealed that the median PFS of patients of lung adenocarcinoma with exon 19 or exon 21 mutation taking afatinib 30 or $40 \mathrm{mg}$ afatinib daily as their initial treatment was 14.5 and 14.8 months, respectively; no significant difference in the PFS was observed between the two groups, and the result was similar to our previous smallscaled study [23].

In terms of OS, the phase 3 LUX-Lung 3 and LUXLung 6 trials reported that the median OS was 31.4 and 33.3 months, respectively. Tanaka et al. performed a 
Table 5 Adverse drug reactions related to different initial afatinib dosages

$\begin{array}{lll}\text { Adverse events } \quad \text { All patients } & \end{array}$

Afatinib $30 \mathrm{mg}$ daily

Maximal grade of events

No

Grade 1

Grade 2

Grade 3

Presence of any moderate and severe ( $\geq$ grade 2 ) adverse events

Details of moderate and severe ( $\geq$ grade 2 ) adverse events

Diarrhea
Stomatitis
Paronychia
Acne and/or skin rash
Dry skin
Pruritus
Adverse events involving skin ${ }^{\text {a }}$
Hepatitis
Interstitial lung disease

Presence of any severe ( $\geq$ grade 3 ) adverse events

Details of severe ( $\geq$ grade 3 ) adverse events

Diarrhea
Stomatitis
Paronychia
Acne and/or skin rash
Dry skin
Pruritus
Adverse events involving skin ${ }^{\text {a }}$
Hepatitis
Interstitial lung disease

\section{Details of adverse events}

Diarrhea

No
Grade 1
Grade 2
Grade 3
Stomatitis
No
Grade 1
Grade 2
Grade 3
Paronychia
No
Grade 1
Grade 2
Grade 3

$\begin{array}{ll}8(4 \%) & 2(2 \%) \\ 62(35 \%) & 50(49 \%) \\ 78(44 \%) & 43(42 \%) \\ 31(17 \%) & 7(7 \%)\end{array}$

$109(61 \%) \quad 50(49 \%)$

$\begin{array}{ll}49(27 \%) & 13(13 \%) \\ 14(8 \%) & 4(4 \%)\end{array}$

$50(28 \%) \quad 29(28 \%)$

$61(34 \%) \quad 17(17 \%)$

$9(5 \%) \quad 1(1 \%)$

$9(5 \%) \quad 1(1 \%)$

$62(35 \%) \quad 18(18 \%)$

$4(2 \%)$

$1(1 \%)$

$0(0 \%)$

$0(0 \%)$

$31(17 \%)$

$7(7 \%)$

$14(8 \%) \quad 2(2 \%)$

$3(2 \%) \quad 2(2 \%)$

$6(3 \%)$

$2(2 \%)$

$11(6 \%)$

$0(0 \%)$

$0(0 \%)$

$0(0 \%)$

$1(1 \%)$

$0(0 \%)$

$11(6 \%)$

$0(0 \%)$

$1(1 \%)$

$1(1 \%)$

$0(0 \%)$

$0(0 \%)$

$\begin{array}{ll}47(26 \%) & 33(32 \%) \\ 83(46 \%) & 56(55 \%) \\ 35(20 \%) & 11(11 \%) \\ 14(8 \%) & 2(2 \%)\end{array}$

125 (70\%)

70 (69\%)

40 (22\%)

$11(6 \%)$

$3(2 \%)$

$28(27 \%)$

$2(2 \%)$

$2(2 \%)$

87 (49\%)

42 (23\%)

44 (25\%)

$6(3 \%)$

49 (48\%)

$24(24 \%)$

27 (26\%)

$2(2 \%)$
Afatinib $40 \mathrm{mg}$ daily

$6(8 \%)$

$12(16 \%)$

35 (45\%)

24 (31\%)

59 (77\%)

0.0002

$36(47 \%)$

$<0.0001$

$10(13 \%)$

0.0253

21 (27\%)

0.8642

44 (57\%)

$8(10 \%)$

$<0.0001$

$8(10 \%)$

0.0043

0.0043

44 (57\%)

$3(4 \%)$

$<0.0001$

0.1913

$0(0 \%)$

24 (31\%)

$<0.0001$

12 (16\%)

0.0008

1 (1\%)

0.7326

$4(5 \%)$

0.2340

11 (14\%)

$<0.0001$

$0(0 \%)$

1 (1\%)

0.2484

$11(14 \%)$

0 (0\%)

$<0.0001$

0.3836

$0(0 \%)$

$<0.0001$

$14(18 \%)$

27 (35\%)

24 (31\%)

$12(16 \%)$

0.0214

55 (71\%)

$12(16 \%)$

$9(12 \%)$

$1(1 \%)$

0.6304
38 (49\%)
$18(23 \%)$
17 (22\%)
$4(5 \%)$

Acne and/or skin rash 
Table 5 Adverse drug reactions related to different initial afatinib dosages (Continued)

\begin{tabular}{|c|c|c|c|c|}
\hline Adverse events & All patients & Afatinib $30 \mathrm{mg}$ daily & Afatinib $40 \mathrm{mg}$ daily & $P$ value \\
\hline No & $34(19 \%)$ & $22(22 \%)$ & $12(16 \%)$ & \\
\hline Grade 1 & $84(47 \%)$ & $63(62 \%)$ & $21(27 \%)$ & \\
\hline Grade 2 & $50(28 \%)$ & $17(17 \%)$ & $33(43 \%)$ & \\
\hline Grade 3 & $11(6 \%)$ & $0(0 \%)$ & $11(14 \%)$ & \\
\hline Dry skin & & & & 0.0171 \\
\hline No & $69(39 \%)$ & $41(40 \%)$ & $28(36 \%)$ & \\
\hline Grade 1 & $101(56 \%)$ & $60(59 \%)$ & $41(53 \%)$ & \\
\hline Grade 2 & $9(5 \%)$ & $1(1 \%)$ & $8(10 \%)$ & \\
\hline Pruritus & & & & 0.0381 \\
\hline No & $112(63 \%)$ & $68(67 \%)$ & $44(57 \%)$ & \\
\hline Grade 1 & $58(32 \%)$ & $33(32 \%)$ & $25(32 \%)$ & \\
\hline Grade 2 & $8(4 \%)$ & $1(1 \%)$ & $7(9 \%)$ & \\
\hline Grade 3 & $1(1 \%)$ & $0(0 \%)$ & $1(1 \%)$ & \\
\hline Adverse events involving skin ${ }^{a}$ & & & & $<0.0001$ \\
\hline No & $24(13 \%)$ & $16(16 \%)$ & $8(10 \%)$ & \\
\hline Grade 1 & $93(52 \%)$ & $68(67 \%)$ & $25(32 \%)$ & \\
\hline Grade 2 & $51(28 \%)$ & $18(18 \%)$ & $33(43 \%)$ & \\
\hline Grade 3 & $11(6 \%)$ & $0(0 \%)$ & $11(14 \%)$ & \\
\hline Hepatitis & & & & 0.1382 \\
\hline No & $172(96 \%)$ & $100(98 \%)$ & $72(94 \%)$ & \\
\hline Grade 1 & $3(2 \%)$ & $1(1 \%)$ & $2(3 \%)$ & \\
\hline Grade 2 & $3(2 \%)$ & $0(0 \%)$ & $3(4 \%)$ & \\
\hline Grade 3 & $1(1 \%)$ & $1(1 \%)$ & $0(0 \%)$ & \\
\hline Interstitial lung disease & & & & 0.2166 \\
\hline Grade 1 & $2(1 \%)$ & $2(2 \%)$ & $0(0 \%)$ & \\
\hline
\end{tabular}

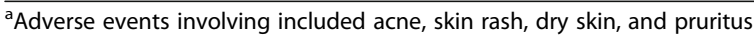

real-world study of first-line afatinib in Japan, showing a median OS of 39.5 months [20]. In the Giotag trial, all patients initially received $40 \mathrm{mg}$ afatinib daily, followed by osimertinib if T790M acquired resistance was reported; the updated median OS was 37.6 months, while the OS was as long as 41.6 months in patients with an exon 19 deletion [30]. In the present multicenter retrospective study, the OS was similar in the $30 \mathrm{mg}$ and $40 \mathrm{mg}$ groups (34.0 and 25.2 months, respectively), suggesting that the clinical effectiveness of $30 \mathrm{mg}$ afatinib daily as the initial treatment was not inferior to $40 \mathrm{mg}$ daily as the starting dose for patients of stage IV lung adenocarcinoma with exon 19 or 21 mutation.

Table 6 Category of afatinib dose reduction

\begin{tabular}{|c|c|c|c|c|}
\hline Variables & All patients & Afatinib $30 \mathrm{mg}$ daily & Afatinib $40 \mathrm{mg}$ daily & $P$ value \\
\hline Dose reduction events & $39(22 \%)$ & $8(8 \%)$ & $31(40 \%)$ & $<0.0001$ \\
\hline \multicolumn{5}{|c|}{ Category of afatinib dose reduction } \\
\hline No change & $140(78 \%)$ & $94(92 \%)$ & $46(60 \%)$ & \\
\hline $40 \mathrm{mg}$ taper down to $30 \mathrm{mg}$ & $22(12 \%)$ & & $22(29 \%)$ & \\
\hline $40 \mathrm{mg}$ taper down to $20 \mathrm{mg}$ & $7(4 \%)$ & & $7(9 \%)$ & \\
\hline $40 \mathrm{mg}$ taper down to $0 \mathrm{mg}$ & $2(1 \%)$ & & $2(3 \%)$ & \\
\hline $30 \mathrm{mg}$ taper down to $20 \mathrm{mg}$ & $1(1 \%)$ & $1(1 \%)$ & & \\
\hline $30 \mathrm{mg}$ taper down to $15 \mathrm{mg}$ & $5(3 \%)$ & $5(5 \%)$ & & \\
\hline $30 \mathrm{mg}$ taper down to $0 \mathrm{mg}$ & $2(1 \%)$ & $2(2 \%)$ & & \\
\hline
\end{tabular}


Table 7 Sites of cancer recurrence in lung adenocarcinoma patients with different initial afatinib dosages

\begin{tabular}{|c|c|c|c|c|}
\hline Recurrence & All patients & Afatinib $30 \mathrm{mg}$ daily & Afatinib $40 \mathrm{mg}$ daily & $P$ value \\
\hline Number of recurrent sites $-n(\%)$ & & & & 0.3222 \\
\hline No recurrence & $89(50 \%)$ & $47(46 \%)$ & $42(55 \%)$ & \\
\hline 1 site & $67(37 \%)$ & $43(42 \%)$ & $24(31 \%)$ & \\
\hline$\geq 2$ sites & $23(13 \%)$ & $12(12 \%)$ & $11(14 \%)$ & \\
\hline \multicolumn{5}{|l|}{ Details of recurrent sites } \\
\hline Central nervous system & $34(19 \%)$ & $18(18 \%)$ & $16(21 \%)$ & 0.5969 \\
\hline Lung & $38(21 \%)$ & $23(23 \%)$ & $15(19 \%)$ & 0.6192 \\
\hline Pleura or pleural effusion & $16(9 \%)$ & $11(11 \%)$ & $5(6 \%)$ & 0.3191 \\
\hline Bone & $16(9 \%)$ & $13(13 \%)$ & $3(4 \%)$ & 0.0399 \\
\hline Liver & $8(4 \%)$ & $5(5 \%)$ & $3(4 \%)$ & 0.7471 \\
\hline Other sites & $7(4 \%)$ & $2(2 \%)$ & $5(6 \%)$ & 0.1214 \\
\hline
\end{tabular}

It should be noted that the current study enrolled patients from May 2014, and only 13 patients (13\%) in the $30 \mathrm{mg}$ group and 5 patients (6\%) in the $40 \mathrm{mg}$ group received osimertinib as the 2nd-line therapy. As osimertinib was very expensive and had not been reimbursed by the Taiwanese National Health Insurance until April 2020 , the majority of patients with acquired-resistance to afatinib chose to receive platinum-based chemotherapy, rather than osimertinib, as their 2nd-line therapy.

The current study found that the predicting factors for poorer PFS might include more metastatic sites, no dose reduction, exon 21, pleural metastasis, and bone metastasis. The predictive factors for poorer OS might include male sex, poorer initial ECOG PS, more metastatic sites, smoking history, bone metastasis, and no adrenal metastasis. Liang et al. suggested that patients with significant pretreatment weight loss ( $>10.0 \%$ in 6 months) had a shorter median PFS, and patients with brain metastases had a poorer ECOG PS status and were associated with a shorter median PFS [31]. Tanaka et al. also showed that patients with dose reduction had a significantly longer PFS than those without dose reduction in a realworld study ( 18.5 vs. 7.9 months, respectively; $p=0.018$ ) [20]. However, the average daily dose of $<20 \mathrm{mg}$ afatinib had a significantly shorter PFS compared with the other higher dose group $(p=0.049)$ [32]. Another study of afatinib in Taiwan showed that OS was not affected by reductions in the afatinib dosage; they also indicated that brain metastases at diagnosis and treatment response to afatinib are two important prognostic factors for OS [33].

Previous clinical trials recommended that $40 \mathrm{mg}$ afatinib daily should be the starting dose in patients whose lung cancer harbors EGFR mutations, however, this dosage was often accompanied by serious ADRs and up to 28 to $53.3 \%$ of patients required dose reduction in the LUX-Lung 3 and LUX-Lung 6 studies [14, 18]. In a realworld study in Japan, $48 \%$ of patients receiving standard
$40 \mathrm{mg}$ afatinib daily had to reduce the dosage and $23 \%$ of patients discontinued treatment due to ADRs [29].

In fact, many clinicians had found more severe ADRs in patients who received standard $40 \mathrm{mg}$ afatinib daily than those who received a 1st generation EGFR TKI, such as gefitinib and erlotinib. There is an urgent need to find a reliable strategy for reducing ADRs associated with afatinib, whilst maintaining its clinical efficacy for the management of lung cancer. Therefore, in clinical practice many clinicians prescribe a lower starting dose of afatinib [22, 23] or perform dose modification $[19,21]$ in order to improve patient outcomes and adherence. Recently, a prospective phase 2 clinical trial, which enrolled 46 patients assessed the efficacy and safety of lower starting doses of afatinib followed by dose modification, according to its toxicity in patients with EGFR mutation-positive NSCLC. The study had a median PFS of 15.2 months (95\% CI: 13.2-not estimable) and the 1year OS rate was $95.6 \%$ (95\% CI: 89.7-100\%) [22].

In a non-interventional, observational study [21] of patients who started with $40 \mathrm{mg}$ afatinib daily, $67.1 \%$ underwent dose reduction, $86.5 \%$ of which occurred in the first 6 months. Dose reductions were more common in the females, East Asian individuals, and those with a lower body weight [21]. A post-marketing, observational study of afatinib in Japan found that a lower starting dose of afatinib was more commonly prescribed to the females and patients with lower body weight [20]. A study by Imai et al. enrolled 40 patients with a median age of 77 years (range, $70-85$ years old) and all of them received $30 \mathrm{mg}$ afatinib as the starting dose; their RR and median PFS were similar to the present study and their ADRs were also acceptable [19].

Since severe ADRs may discontinue the use of afatinib or lead to dose reduction, one must pay close attention to the incidence and severity of ADRs during the treatment of lung adenocarcinoma harboring exon 19 or exon 21 mutations. Of patients who received $40 \mathrm{mg}$ 
afatinib daily as their starting dose in the phase 3 LUXLung 3 and LUX-Lung 6 trials, 73.0 and 80.6\%, respectively experienced grade 3 or higher treatment-related ADRs; the incidence of ADRs dropped to 11.9 and $20.5 \%$, respectively after the dosage was reduced $[13,14$, 18]. In a real-world study, grade 3 or higher ADRs occurred in $30.4 \%$ of patients [20]. The present study demonstrated that acne and/or skin rash, diarrhea, dry skin, and paronychia were common ADRs and significantly fewer events were observed in the $30 \mathrm{mg}$ group than in the $40 \mathrm{mg}$ group, similar to the findings of our previous study [23]. Furthermore, patients who received $30 \mathrm{mg}$ afatinib daily had a significantly lower incidence of severe ADRs than those receiving $40 \mathrm{mg}$ daily, in terms of diarrhea, and acne and/or rash. Fewer severe ADRs might be associated with better drug compliance and a better overall quality of life for the patients. Besides, there was no increased incidence of recurrent central nervous system metastasis in patients receiving $30 \mathrm{mg}$ daily afatinib as the starting dose compared with those receiving $40 \mathrm{mg}$ daily initially in the current study.

Our study still had some limitations. Firstly, although the study enrolled patients from three hospitals, the retrospective design of this study might make the results less reliable than other standard prospective clinical trials. Secondly, the number of cases enrolled in the study was relatively low for a retrospective study. However, this study enrolled the largest number of patients receiving $30 \mathrm{mg}$ afatinib daily as the starting dose to date. Thirdly, patients with recurrent lung cancer were excluded from the current study. Fourthly, we only enrolled lung adenocarcinoma patients and excluded those with squamous cell carcinoma or other rare types of lung cancer. Almost 99\% of residents in Taiwan are covered by the Taiwan National Health Insurance and only adenocarcinoma harboring susceptible EGFR mutations is reimbursed. To obtain a more homogenous patient cohorts for investigating the factors associated with PFS and OS, we included only those initially diagnosed with stage IV lung adenocarcinoma in the current study. Fifthly, only $13 \%$ of patients in the $30 \mathrm{mg}$ group and $6 \%$ of patients in the $40 \mathrm{mg}$ group received osimertinib as their 2nd-line therapy. Most patients might receive a platinum-based chemotherapy, rather than osimertinib, as a salvage therapy because osimertinib had not been reimbursed by the National Health Insurance in Taiwan until April 2020. Further study is warranted to understand the effect of different 2nd-ling therapy on OS in patients receiving 1st-line afatinib for their lung adenocarcinoma harboring EGFR mutation.

In conclusion, a lower starting dose (30 mg daily) of afatinib for patients of lung adenocarcinoma harboring susceptible EGFR mutations showed similar RR, PFS, and OS compared with those receiving a standard $40 \mathrm{mg}$ daily as the initial dose of afatinib. The lower starting dose was associated with fewer ADRs, as well as fewer moderate and severe ADRs. A further large-scale prospective trial is urgently needed to confirm these findings.

\section{Abbreviations \\ NSCLC: Non-small cell lung cancer; ORR: Objective response rate; OS: Overall survival; PFS: Progress free survival; HR: Hazard ratio; RR: Risk ratio; \\ ADR: Adverse drug reaction; HR: Hazard ratios; Cis: Confidence intervals; \\ EGFR: Epidermal growth factor receptor; TKI: Tyrosine kinase inhibitor; ARMS \\ : Amplification refractory mutation specific; PCRs: Polymerase chain reactions; \\ ECOG: Eastern Cooperative Oncology Group; PS : Performance status; TTF-1 \\ : Thyroid transcription factor-1; PDL-1 : Programmed death-ligand 1; RECl \\ ST: Response Evaluation Criteria in Solid Tumors}

\section{Acknowledgements}

The authors would like to thank all the hospital staff, the lung cancer teams and the patients in Kaohsiung Medical University Hospital, Kaohsiung

Municipal Ta-Tung Hospital and Kaohsiung Municipal Siaokang Hospital in Taiwan.

\section{Authors' contributions}

The paper was conceived and designed by YC Chen, MJ Tsai and CJ Yang and contributed to by all the authors. JY Hung and MS Huang and IW

Chong were as consultants and provided many valuable comments. MH Lee, CY Kuo, MC Shen and YM Tsai performed the interviews and conducted the qualitative content analysis. YC Chen, HC Chen, CJ Yang wrote the first draft of the manuscript, which was critically reviewed, revised, and approved for submission by all the authors.

\section{Funding}

This work was supported by Kaohsiung Medical University, Kaohsiung Medical University Hospital, Kaohsiung Municipal Ta-Tung Hospital and Kaohsiung Municipal Siaokang Hospital in Taiwan.

\section{Availability of data and materials}

The datasets used and/or analyzed during the current study are available from the corresponding author on a reasonable request.

\section{Declarations}

Ethics approval and consent to participate

The Institutional Review Board of Kaohsiung Medical University Hospital (KMUH) approved the current study (approval no. KMUHIRB-E (II)-20,150,162). Patient records were anonymized and de-identified prior to all analyses. Waived the need for written informed consent due to a retrospective entity.

Consent for publication

not applicable.

\section{Competing interests}

The authors declare no conflict of interest.

\footnotetext{
Author details

'Department of Pharmacy, Kaohsiung Municipal Ta-Tung Hospital, Kaohsiung Medical University, Kaohsiung, Taiwan. ${ }^{2}$ Graduate Institute of Medicine, College of Medicine, Kaohsiung Medical University, Kaohsiung, Taiwan. ${ }^{3}$ Division of Pulmonary and Critical Care Medicine, Kaohsiung Medical University Hospital, Kaohsiung Medical University, Kaohsiung, Taiwan. ${ }^{4}$ Faculty of Medicine, Kaohsiung Medical University, Kaohsiung, Taiwan. ${ }^{5}$ Department of Internal Medicine, Kaohsiung Municipal Siaogang Hospital, Kaohsiung Medical University, Kaohsiung, Taiwan. ${ }^{6}$ Department of Pharmacy, Kaohsiung Medical University Hospital, Kaohsiung Medical University, Kaohsiung, Taiwan. ${ }^{7}$ Department of Internal Medicine, E-DA Cancer Hospital, Kaohsiung, Taiwan. ${ }^{8}$ Respiratory therapy, College of Medicine, Kaohsiung Medical University, Kaohsiung, Taiwan. ${ }^{9}$ Department of Internal Medicine, Kaohsiung Medical University Hospital, No. 100, Tzyou First Road, Kaohsiung City, Taiwan.
} 


\section{Received: 23 September 2020 Accepted: 22 April 2021} Published online: 03 May 2021

\section{References}

1. Marino P, Pampallona S, Preatoni A, Cantoni A, Invernizzi F. Chemotherapy vs supportive care in advanced non-small-cell lung cancer. Results of a meta-analysis of the literature. Chest. 1994;106(3):861-5. https://doi.org/1 0.1378/chest.106.3.861

2. Scagliotti GV, Parikh P, von Pawel J, Biesma B, Vansteenkiste J, Manegold C, et al. Phase III study comparing cisplatin plus gemcitabine with cisplatin plus pemetrexed in chemotherapy-naive patients with advanced-stage nonsmall-cell lung cancer. J Clin Oncol. 2008;26(21):3543-51. https://doi.org/1 0.1200/JCO.2007.15.0375

3. Schiller JH, Harrington D, Belani CP, Langer C, Sandler A, Krook J, et al. Comparison of four chemotherapy regimens for advanced non-small-cell lung cancer. N Engl J Med. 2002;346(2):92-8. https://doi.org/10.1056/ NEJMoa011954.

4. Huang MS, Tsai JR, Shen MC, Chou SH, Yang CJ. Pemetrexed as a possible cause of severe rhabdomyolysis in the treatment of lung cancer. Lung Cancer. 2012;76(3):491-2. https://doi.org/10.1016/j.lungcan.2012.02.009.

5. Greenhalgh J, Dwan K, Boland A, Bates V, Vecchio F, Dundar Y, et al. Firstline treatment of advanced epidermal growth factor receptor (EGFR) mutation positive non-squamous non-small cell lung cancer. Cochrane Database Syst Rev. 2016;5:CD010383.

6. Ding PN, Lord SJ, Gebski V, Links M, Bray V, Gralla RJ, et al. Risk of treatmentrelated toxicities from EGFR tyrosine kinase inhibitors: a meta-analysis of clinical trials of Gefitinib, Erlotinib, and Afatinib in advanced EGFR-mutated non-small cell lung Cancer. J Thorac Oncol. 2017;12(4):633-43. https://doi. org/10.1016/j.jtho.2016.11.2236.

7. Maemondo M, Inoue A, Kobayashi K, Sugawara S, Oizumi S, Isobe H, et al. Gefitinib or chemotherapy for non-small-cell lung cancer with mutated EGFR. N Engl J Med. 2010;362(25):2380-8. https://doi.org/10.1056/NEJMoa 0909530.

8. Mitsudomi T, Morita S, Yatabe Y, Negoro S, Okamoto I, Tsurutani J, et al. Gefitinib versus cisplatin plus docetaxel in patients with non-small-cell lung cancer harbouring mutations of the epidermal growth factor receptor (WJTOG3405): an open label, randomised phase 3 trial. Lancet Oncol. 2010; 11(2):121-8. https://doi.org/10.1016/S1470-2045(09)70364-X.

9. Mok TS, Wu YL, Thongprasert S, Yang CH, Chu DT, Saijo N, et al. Gefitinib or carboplatin-paclitaxel in pulmonary adenocarcinoma. N Engl J Med. 2009; 361(10):947-57. https://doi.org/10.1056/NEJMoa0810699.

10. Rosell R, Carcereny E, Gervais R, Vergnenegre A, Massuti B, Felip E, et al. Erlotinib versus standard chemotherapy as first-line treatment for European patients with advanced EGFR mutation-positive non-small-cell lung cancer (EURTAC): a multicentre, open-label, randomised phase 3 trial. Lancet Oncol. 2012;13(3):239-46. https://doi.org/10.1016/S1470-2045(11)70393-X.

11. Zhou C, Wu YL, Chen G, Feng J, Liu XQ, Wang C, et al. Erlotinib versus chemotherapy as first-line treatment for patients with advanced EGFR mutation-positive non-small-cell lung cancer (OPTIMAL, CTONG-0802): a multicentre, open-label, randomised, phase 3 study. Lancet Oncol. 2011; 12(8):735-42. https://doi.org/10.1016/S1470-2045(11)70184-X.

12. Solca F, Dahl G, Zoephel A, Bader G, Sanderson M, Klein C, et al. Target binding properties and cellular activity of afatinib (BIBW 2992), an irreversible ErbB family blocker. J Pharmacol Exp Ther. 2012;343(2):342-50. https://doi.org/10.1124/jpet.112.197756.

13. Sequist LV, Yang JC, Yamamoto N, O'Byrne K, Hirsh V, Mok T, et al. Phase III study of afatinib or cisplatin plus pemetrexed in patients with metastatic lung adenocarcinoma with EGFR mutations. J Clin Oncol. 2013;31(27):332734. https://doi.org/10.1200/JCO.2012.44.2806.

14. Wu YL, Zhou C, Hu CP, Feng J, Lu S, Huang Y, et al. Afatinib versus cisplatin plus gemcitabine for first-line treatment of Asian patients with advanced non-small-cell lung cancer harbouring EGFR mutations (LUX-lung 6): an open-label, randomised phase 3 trial. Lancet Oncol. 2014;15(2):213-22. https://doi.org/10.1016/S1470-2045(13)70604-1.

15. Yang JC, Wu YL, Schuler M, Sebastian M, Popat S, Yamamoto N, et al. Afatinib versus cisplatin-based chemotherapy for EGFR mutation-positive lung adenocarcinoma (LUX-lung 3 and LUX-lung 6): analysis of overall survival data from two randomised, phase 3 trials. Lancet Oncol. 2015;16(2): 141-51. https://doi.org/10.1016/S1470-2045(14)71173-8.

16. Park K, Tan EH, O'Byrne K, Zhang L, Boyer M, Mok T, et al. Afatinib versus gefitinib as first-line treatment of patients with EGFR mutation-positive non- small-cell lung cancer (LUX-lung 7): a phase 2B, open-label, randomised controlled trial. Lancet Oncol. 2016;17(5):577-89. https://doi.org/10.1016/S14 70-2045(16)30033-X.

17. Takeda M, Okamoto I, Nakagawa K. Pooled safety analysis of EGFR-TKI treatment for EGFR mutation-positive non-small cell lung cancer. Lung Cancer. 2015;88(1):74-9. https://doi.org/10.1016/j.lungcan.2015.01.026.

18. Yang JC, Sequist LV, Zhou C, Schuler M, Geater SL, Mok T, et al. Effect of dose adjustment on the safety and efficacy of afatinib for EGFR mutationpositive lung adenocarcinoma: post hoc analyses of the randomized LUXlung 3 and 6 trials. Ann Oncol. 2016;27(11):2103-10. https://doi.org/10.1 093/annonc/mdw322

19. Imai H, Kaira K, Suzuki K, Anzai M, Tsuda T, Ishizuka T, et al. A phase II study of afatinib treatment for elderly patients with previously untreated advanced non-small-cell lung cancer harboring EGFR mutations. Lung Cancer. 2018;126:41-7. https://doi.org/10.1016/j.lungcan.2018.10.014.

20. Tanaka H, Taima K, Itoga M, Ishioka Y, Baba K, Shiratori T, et al. Real-world study of afatinib in first-line or re-challenge settings for patients with EGFR mutant non-small cell lung cancer. Med Oncol. 2019;36(6):57. https://doi. org/10.1007/s12032-019-1278-9.

21. Halmos B, Tan EH, Soo RA, Cadranel J, Lee MK, Foucher P, et al. Impact of afatinib dose modification on safety and effectiveness in patients with EGFR mutation-positive advanced NSCLC: results from a global real-world study (RealGiDo). Lung Cancer. 2019;127:103-11. https://doi.org/10.1016/j.lungca n.2018.10.028.

22. Yokoyama T, Yoshioka H, Fujimoto D, Demura Y, Hirano K, Kawai T, et al. A phase II study of low starting dose of afatinib as first-line treatment in patients with EGFR mutation-positive non-small-cell lung cancer (KTORG1402). Lung Cancer. 2019;135:175-80. https://doi.org/10.1016/j. lungcan.2019.03.030.

23. Yang CJ, Tsai MJ, Hung JY, Lee MH, Tsai YM, Tsai YC, et al. The clinical efficacy of Afatinib $30 \mathrm{mg}$ daily as starting dose may not be inferior to Afatinib $40 \mathrm{mg}$ daily in patients with stage IV lung adenocarcinoma harboring exon 19 or exon 21 mutations. BMC Pharmacol Toxicol. 2017; 18(1):82. https://doi.org/10.1186/s40360-017-0190-1.

24. Tsai MJ, Hung JY, Lee MH, Kuo CY, Tsai YC, Tsai YM, et al. Better Progression-Free Survival in Elderly Patients with Stage IV Lung Adenocarcinoma Harboring Uncommon Epidermal Growth Factor Receptor Mutations Treated with the First-line Tyrosine Kinase Inhibitors. Cancers (Basel). 2018;10(11):434.

25. Yang CJ, Hung JY, Tsai MJ, Wu KL, Liu TC, Chou SH, et al. The salvage therapy in lung adenocarcinoma initially harbored susceptible EGFR mutation and acquired resistance occurred to the first-line gefitinib and second-line cytotoxic chemotherapy. BMC Pharmacol Toxicol. 2017;18(1):21. https://doi.org/10.1186/s40360-017-0130-0.

26. Yang CJ, Tsai MJ, Hung JY, Liu TC, Chou SH, Lee JY, et al. Pemetrexed had significantly better clinical efficacy in patients with stage IV lung adenocarcinoma with susceptible EGFR mutations receiving platinum-based chemotherapy after developing resistance to the first-line gefitinib treatment. Onco Targets Ther. 2016;9:1579-87. https://doi.org/10.2147/OTT. S100164.

27. Yang CJ, Tsai MJ, Hung JY, Tsai YM, Lee JY, Chou SH, et al. Poorer prognosis in Taiwanese female ever smokers with stage IV lung adenocarcinoma who were readministered a tyrosine kinase inhibitor. Onco Targets Ther. 2016;9: 1511-8. https://doi.org/10.2147/OTT.S100169.

28. Liu CY, Wang CL, Li SH, Hsu PC, Chen CH, Lin TY, et al. The efficacy of 40 mg versus dose de-escalation to less than $40 \mathrm{mg}$ of afatinib (Giotrif) as the first-line therapy for patients with primary lung adenocarcinoma harboring favorable epidermal growth factor mutations. Oncotarget. 2017;8(57):9760212. https://doi.org/10.18632/oncotarget.18746.

29. Tamura K, Nukiwa T, Gemma A, Yamamoto N, Mizushima M, Ochai K, et al. Real-world treatment of over 1600 Japanese patients with EGFR mutationpositive non-small cell lung cancer with daily afatinib. Int J Clin Oncol. 2019; 24(8):917-26. https://doi.org/10.1007/s10147-019-01439-5.

30. Hochmair MJ, Morabito A, Hao D, Yang CT, Soo RA, Yang JC, et al. Sequential afatinib and osimertinib in patients with EGFR mutation-positive non-small-cell lung cancer: updated analysis of the observational GioTag study. Future Oncol. 2019;15(25):2905-14. https://doi.org/10.2217/fon-2019-0346.

31. Liang SK, Hsieh MS, Lee MR, Keng LT, Ko JC, Shih JY. Real-world experience of afatinib as a first-line therapy for advanced EGFR mutation-positive lung adenocarcinoma. Oncotarget. 2017;8(52):90430-43. https://doi.org/10.18632/ oncotarget.19563. 
32. Lim CK, Wei YF, Tsai MS, Chen KY, Shih JY, Yu CJ. Treatment effectiveness and tolerability of afatinib at different doses in patients with EGFR-mutated lung adenocarcinoma: how low can we go? Eur J Cancer. 2018;103:32-40. https://doi.org/10.1016/j.ejca.2018.07.128.

33. Liang SK, Lee MR, Liao WY, Ho CC, Ko JC, Shih JY. Prognostic factors of afatinib as a first-line therapy for advanced EGFR mutation-positive lung adenocarcinoma: a real-world, large cohort study. Oncotarget. 2018;9(34): 23749-60. https://doi.org/10.18632/oncotarget.25255.

\section{Publisher's Note}

Springer Nature remains neutral with regard to jurisdictional claims in published maps and institutional affiliations.

Ready to submit your research? Choose BMC and benefit from:

- fast, convenient online submission

- thorough peer review by experienced researchers in your field

- rapid publication on acceptance

- support for research data, including large and complex data types

- gold Open Access which fosters wider collaboration and increased citations

- maximum visibility for your research: over $100 \mathrm{M}$ website views per year

At $\mathrm{BMC}$, research is always in progress.

Learn more biomedcentral.com/submissions 